\title{
The Application of Quantum Theory to Explain the Effects of Various Management Practices on Basic Research
}

Management of research involves myriad functions of nonresearch character that nevertheless influence the enterprise greatly. They generally fall in one of two categories: the organization of the process and the evaluation of its results. Over much of the past half century, the management was benevolent and the managers enlightened, for the most part. Of late, pressures beyond the control of our benefactors and beyond the scope of this article have mitigated in favor of bruteforce one-size-fits-all organization and evaluation criteria. ${ }^{1}$ What the perpetrators of this change have overlooked is the fundamental stochastic nature of basic research ${ }^{2}$ and the miraculous way it mimics a quantized system of weakly interacting researchers.

First let's look at the impact of organizational overlays on research. The least visionary approach to organization relies on categorization. If a project doesn't fit neatly into a well-defined category, well separated from all others, it will disappear. Quantum confinement forces projects out of the continuum of creative research into the well-separated, very stationary states of deep potential wells. Some hybridization of orbitals may contribute to short-range mingling among researchers, but as any researcher worth her wave function knows, the broader the width of her state, the shorter lived it will be. Worse yet, just as the real component of the phase of any wave function damps its expanse, the injection of reality requirements into research agenda severely hampers tunneling exchanges among neighboring disciplinary potential wells.

Evaluation of research has quantum consequences too. We know some jobs are done better than others. We would prefer that those working for us do good jobs. We ought to have a way to determine how well a job is done. That seems trivially obvious if we're repairing the kitchen sink, building a bridge on time and on budget, or rescuing the citizenry of a distant land from famine and pestilence. But scientific research is a different story. Asking a basic researcher how his particular project will contribute to industrial competitiveness, national security, quality of life, etc., is like asking one gas molecule when and how much it plans to contribute to a vessel's pressure on its next collision with a wall.

Worse yet, as we all know, the act of measurement alters the system being measured. The research project that had been going along nicely as a linear combination of its chosen basis states is forced into an eigenstate of the joint research-plus-evaluation system, distorting its original intent and misleading evaluators. Only a dwindling number of hidden variables, otherwise known as discretionary funds, are left to preserve the essential nature of pure inquiry in the face of a not-so-hidden political agenda of this generation of demanding benefactors.

Faced with an impossible task, evaluators use short-term anecdotal second guessing or, in quantum parlance, second quantization into the smallest evaluative units (seu). (And seu, by the time it rises to upper management, becomes an account number in a cost center. In the press, it's the familiar sound bite or column inch. And in Congress, it is a line item or an earmark.) Creation and annihilation operators in this parameterization can put an embryonic project on the front page (witness cold fusion), can defund major programs (à la the SSC), or can indulge in vacuum polarization and virtually create and destroy a project on the drawing board (the ANS comes to mind). CPT invariance for this system is in grave doubt. There are charges (C) of excessive indirect charges $(C)$. There is no parity $(P)$ in pork $(\mathrm{P})$. And time reversal invariance (T) is being sorely tested by threats of rescission. The only basic principle certain to be preserved is the uncertainty principle - the results of basic research will continue to be as uncertain as next year's budget and neither party's platform includes a constant Plan(c)k for science.

Although it runs counter to the fiercely independent fermion-like nature of noncommuting researchers, many suggest that a collective response is required-a phase transition for a tran- sitional phase. As painful as it might be, it behooves researchers to band together and commute to Washington after undergoing symmetry-change operations. They can then condense into a united nondissipative special-interest lobby of born-again bosons and add their integral spin to the research message. In this compact state they should be able to deliver the message of basic research without exceeding the attention span of Congress. Based on the recent State-of-the-Union address that was lambasted for using a full 80 minutes to discuss the entire United States of America's plans in all areas of interest for an entire year, we estimate that our message dare not exceed one seu or about 15 seconds on the evening news.

If enough policymakers tune in, we are virtually assured that a new era will dawn where destructive interference and perturbations of the first order give way to complementarity and many one-hand-washes-the-other interactions in reciprocal space. However, if the quantum nature of research does not regain respect, its expectation value for a large cross section of researchers is very poor indeed.

E.N. KaUFMANN

1. Senator Barbara Mikulski on Public Radio (WAMU, Washington, D.C. on January 11, 1995) warned that "politics is going to drive science, and it's going to drive it through the cutting process, unless science can articulate a clear vision about what it wants to accomplish."

2. Neal Lane, Director of the National Science Foundation, in the House Science Committee testimony on January 6, 1995, declared, "NSF support of research focuses almost exclusively on answers to fundamental questions that defy our ability to predict the outcomes." Congressman Robert Walker on Public Radio (op. cit.) conceded that "no one knew that [research on bread mold would ultimately produce penicillin] at the time that the research began." Last August the Administration released the report Science in the National Interest that includes the acknowledgment,

"Fundamental science is often the wellspring of advanced technology in ways that are completely unpredictable. 